\title{
An efficient algorithm for computer tomography in low radiation images
}

\author{
B.S. Sathishkumar ${ }^{1 *}$, G. Nagarajan ${ }^{2}$ \\ ${ }^{1}$ Department of ECE, Research Scholar, Pondicherry University, Pondicherry Engineering College, Pondicherry, India \\ ${ }^{2}$ Department of ECE, Professor, Pondicherry University Pondicherry Engineering College, Pondicherry, India
}

Corresponding Author Email: bssathishkumar.79@gmail.com

https://doi.org/10.18280/ama_a.610403

Received: 9 June 2018

Accepted: 15 October 2018

\section{Keywords:}

computer tomography, fan beam tomography, image reconstruction, iterative algorithm, parallel beam tomography, radiation dose

\begin{abstract}
Computer Tomography (CT) is one of the efficient imaging techniques employed in medical field for the past few decades. In CT Scanning, image quality has influenced by several factors like noise, slice thickness, minimum and maximum contrast resolution, radiation dose etc., Radiation dose is one among important and challenging issues taken to optimize the reconstruction algorithm in CT. The radiation dose is controlled by tube current*time product (mAs), pitch or table speed, slice thickness, beam energy (kVp) and number of patients. In this paper, Landweber algorithm is used to check the improvement in quality of image in low radiation dose. The projected algorithm is compared with existing iterative reconstruction algorithm in Test Phantom, Head image and Thorax image and shows better results. The proposed methods will be useful to optimize an iterative reconstruction algorithm with adequate level of quality in computer tomography.
\end{abstract}

\section{INTRODUCTION}

Computer Tomography is a superior imaging method which uses an ionizing radiation and algorithms to attain the sectional view of object with higher accuracy in the variety of disease entity. Due to the benefits ensured in CT, its usage in medical field is more distinguished with other imaging [1]. Major issues in $\mathrm{CT}$ are needed to minimize the X-ray radiation during the imaging procedures. Advantage of using dual tree complex wavelet transform is that instead of capturing the signal energy in different path [2]. Necessity of preprocessing methods are for image normalization and to increase the contrast for achieving accurate analysis [3]. Under the situation of scarcity of data in the target domain, the performance of the traditional agent simulation model tends to decrease. In this scenario, the useful knowledge in the source domain is extracted to guide the target domain learning to obtain more appropriate class information and agent simulation performance is an effective learning method [4].

The patient exposed in X-ray radiation which may create the biological effects like cancer hereditary effects and noncancer effect like respiration diseases, stroke and digestive disorders. Radiation dose is challenging are in image quality and diagnostic accuracy [5]. Increasing the radiation dose may reduce the noise and improve the spatial resolution in the image. But it increases the risk to patients. While decrease the radiation dose may lead more noise and lack of diagnostic accuracy in CT [6]. CT produces more accuracy in image. Many procedures to reduce the radiation dose are followed while maintaining adequate quality of image in clinical task [7].

The result of dose reduction can be approached in two conditions: The first one is to appropriately define the target image quality for each specific diagnostic task. The second one, dose reduction is to improve some aspects of image quality, such as reducing image noise which can then be implemented in order to allow radiation dose reduction [8]. This task can be performed by optimizing scanning techniques, improving the image reconstruction and data processing [9]. Radiation dose is derived in many ways. The scanner radiation output is represented by volume CT dose index $\left(\mathrm{CTDI}_{\mathrm{Vol}}\right)$. Organ dose is the measure of radiation risk to the organ undergoing in CT. Radiation dose also depends on time and sex. Effective dose can be defined equivalent doses in tissues and organs [10].

The paper is organized as follows. The section II discuss about the previous related works in this area. Section III explains about the proposed methods of algorithm and other iterative algorithms. Section IV discuss about the simulation results and their interpretation. Section V concludes the work.

\section{RELATED WORKS}

There are many algorithms reported to improve the quality of image during reconstruction in case of normal and reduced dose level. Image reconstruction algorithm is one which is more active role in achieving good quality of image to extract the detailed information from image. Two major types of reconstruction methods are used in tomography. Analytical is a single step reconstruction and it is quite sophisticated in more projections with less error. Iterative algorithm has best solution in the case of less number of projections and also noisy. Fig: 1. shows the block diagram of image reconstruction technique. Although the considerable computational complexity and speed of iterative reconstruction algorithm, required more memory space and dealing with real time data are major challenging issues in practical implementations.

It is seen that real-world problems are tainted with uncertainty due to lack of knowledge, imprecision, vagueness 
etc [11]. The sufficient conditions for determining the nonsingular H-matrices were given by applying the theory of diagonally dominant matrix [12]. This article introduces its important theoretical foundations and classifies and summarizes the latest research progress of error-eliminating theories [13].
Many imaging strategies can reduce radiation dose while maintain appropriate diagnostic quality for the clinical task [14]. Dose Reduction Guidance (DRG) is an alternative tool to assist in building protocol that utilize Adaptive Statistical image Reconstruction (ASiR) to reduce the Mas needed for scan acquisition [15].

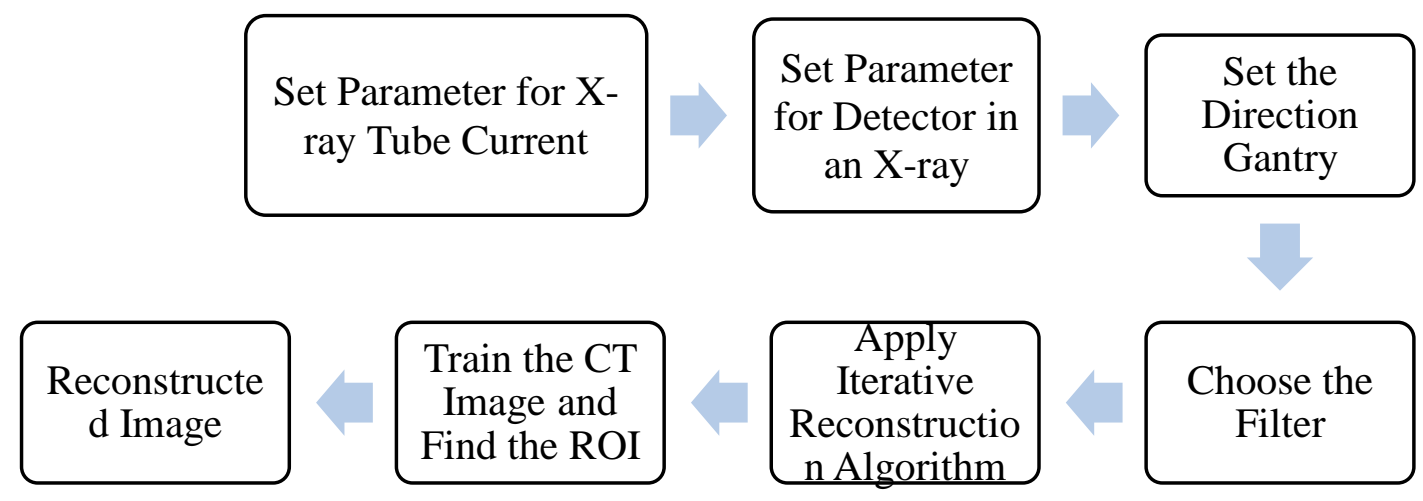

Figure 1. Block diagram of image reconstruction technique

Aaron Sodickson [16], demonstrated the hardware and software available to appropriate quality in a radiation exposure for the clinical scenario. In CT, Artifacts have different from noise, beam hardening, scattering, motion, helical, ring, etc., F. E. Boas, et al., [17] presented the reduction of artifacts and methods to avoid the artifacts in tomography. CT scanners used to create cross-sectional images through attenuation properties from different directions. The author [18] proposed radiation reduction strategies without compromising the quality of reconstructed image. Huaiqun Guany, et al., [19] made a survey on various projections methods in the computer tomography were described.

The two aspects of a contradiction exist in the same system and can mutually transform under certain conditions [20]. It focuses on hiding secret messages inside a cover medium [21]. All of the techniques discussed are reversible information embedding techniques in the spatial domain [22]. This paper presents an approach to simplify the feature extraction method to identify varying character patterns [23].

Various dose reduction and patient movements during inspections are investigated in tomography [24-25]. W. C. Scarfe, et al., [26] presents developments and usage of the cone beam in clinical applications. CBCT is an accurate image modality in normal and reduced radiation dose in proving the quality of image. Also, the future enhancement in $\mathrm{CBCT}$ is presented like reducing scan time, provide multimodal imaging and improving image fidelity. [27] Described the mathematical and benefits of radon transform in the development of image reconstruction in CT image.

\subsection{Dose reduction stratégies}

The quality of image is highly suffered by noise and inversely related to radiation energy. This shows that the dose reduction is important while try to reduce patient dose, image quality should not be compromised. The challenge is in finding a balance between dose and noise that allows the images to be of diagnostic quality while utilizing the lowest dose possible. Noise (specifically, quantum noise) is generally characterized by graininess on image. The way to reduce the noise is to increasing the radiation dose. Since radiation dose is inversely proportional to image noise. In other words, noise is inversely related to the number of $\mathrm{X}$ rays (which are proportional to $\mathrm{mAs}$ ) used to create the image by the following relationships (assuming all other parameters are kept constant):

Noise $\alpha \frac{1}{\text { Number of } x \text {-rays }} \alpha \frac{1}{m A s} \alpha \frac{1}{\text { Radiation Dose }}$

Hence the selection of optimal parameters in the dose reduction is needed. Dose reduction parameters are classified as follows in Fig: 2.

$>$ Scanning Parameters

$>$ Anatomical Parameters

$>$ Technical Parameters

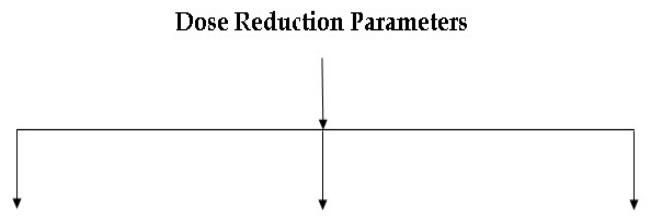

$\begin{array}{lcc}\text { Scanning Parameters } & \text { Anatomical Parameters } & \text { Technical Parameters } \\ \text { - Scanning Geometry } & \text { - Weight } & \text { - Beam Collimation } \\ \text { - Tube Current } & \text { - Cross Sectional } & \text { - Beam Filtration } \\ \text { - Tube Potential } & \text { Dimensions } & \text { - Automated Tube } \\ \text { - Scanning Mode } & & \text { Current Modulation } \\ \text { - Scanning Length } & & \text { - Projection Adaptive } \\ \text { - Collimation Pitch } & & \text { Reconstruction Filters } \\ \text { - Gantry Rotation Time } & & \text { - Noise }\end{array}$

Figure 2. Clasificación of dose reduction 


\section{PROPOSED ALGORITHM}

This work is to implement an efficient algorithm for computer tomography in low radiation CT images. There are many efforts and investigate in improvement in quality of image, technology and image reconstruction algorithm to achieve the tradeoff between image quality and radiation dose. There are many efforts have been taken on suitable utilization and safety of CT scanning to make the better quality of image in reducing the radiation dose. There are more efforts and investigations in improvement in quality of image, technology and image reconstruction algorithm to achieve the tradeoff between image quality and radiation dose.

\subsection{Reconstruction algorithms}

Image reconstruction is aided to improve the differentiation of contrast. The number of projections is fixed to reduce dose level and scanning time to the patient and then the information received from the projections improved by high performance reconstruction algorithm. The varieties of algorithms are available to produce accurate reconstruction at the cost high computation time in CT. Some of the algorithms uses the prior knowledge about the object like number of different kind of materials, it characteristics and gray levels, etc., these parameters potentially effective in selection of the projections, increases the accuracy and significantly reduce the noise.

The Landweber algorithm for solving the system $A x=B$ is

$$
x^{k+1}=x^{k}+\gamma A \dagger\left(b-A x^{k}\right)
$$

where, $\gamma$ is a selection parameter, we can write the Landweber iteration as

$$
\begin{aligned}
& x^{k+1}=T x^{k} \\
& \mathrm{Tx}=(1-\gamma A \dagger A) \mathrm{x}+\mathrm{A} \dagger b=B x+b
\end{aligned}
$$

The Landweber algorithm actually solve the square linear system $A \dagger A=\mathrm{A} \dagger b$ for a least-square solution of $\mathrm{Ax}=\mathrm{B}$ for the unique solution, of $\mathrm{Ax}=\mathrm{B}$, say $\hat{x}$ the error at the $k^{t h}$ step is

$$
e^{k}=\hat{x}-x^{k} \text { and } \mathrm{B} e^{k}=e^{k+1}
$$

When there is multiple solution of $A x=b$ the solution fixed by Landweber algorithm will be the one of the closest to the starting vector.

\section{SIMULATION RESULTS}

The proposed scheme is simulated in the MATLAB. The parameters like Correlation coefficient Vs number of iteration and Mean squared error Vs number of iterations are evaluated in reduced dose level in computer tomography performed through different iterative algorithms. The proposed landweber iteration algorithm and other iterative algorithms performance are compared in terms of $\mathrm{SC}, \mathrm{AD}$, MD, NAE, PSNR., etc. these parameters are found in parallel beam and Fan beam geometry arrangements.

To reduce the exposure of radiation to the patient in computer tomography, small number of projections has been explored. (e.g., projections restricted to a small region of interest). With small set of orientations, the tomographic image is reconstructed by algorithm like SART, DART, CAV, Cimmino and Landweber. Also set the parameters initially at the lower the tube current which inturn reduce the radiation exposure of less amount. The number of projections is taken as small. This gives an image which consists of fewer amounts of image details. The parameter such as Mean Absolute error and correlation coefficient are measured with different iteration in test image, head image and thorax image simulated through different iterative algorithms. The simulation is done to obtain the correlation coefficient versus number of iterations and Mean square error versus number of iteration curves are simulated for the reduced dose in parallel beam and fan beam type of computer tomography and compared with the different iterative algorithm. A MATLAB generated test phantom, Head CT and Thorax images are considered as input image and reconstructed image of different algoeithms for fixed number of projections and iteration shows in Figure 3.
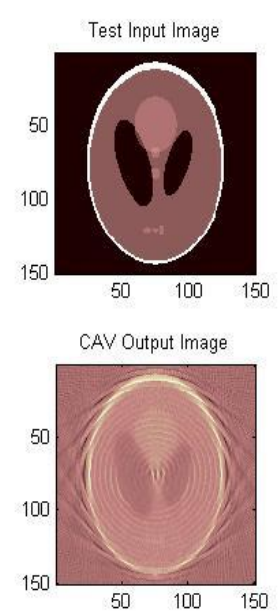
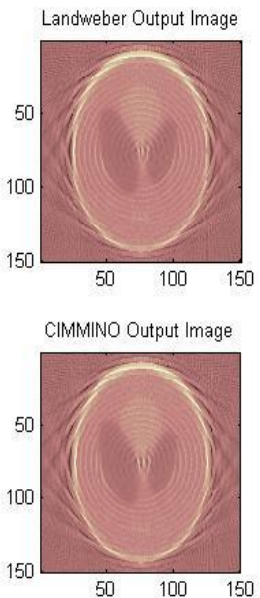

(a)
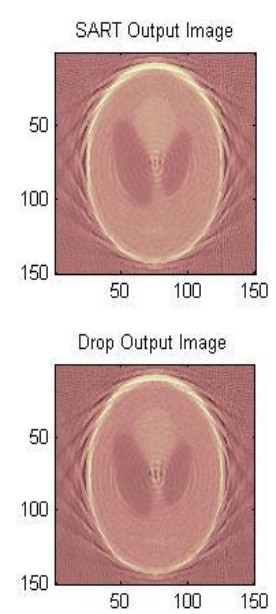

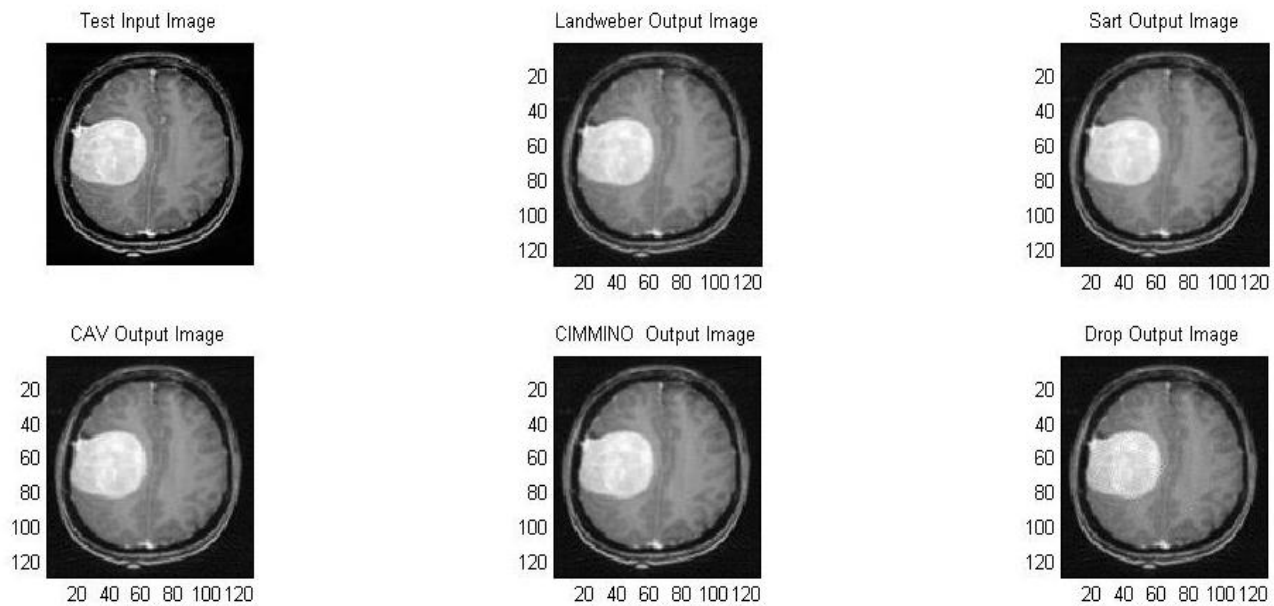

(b)
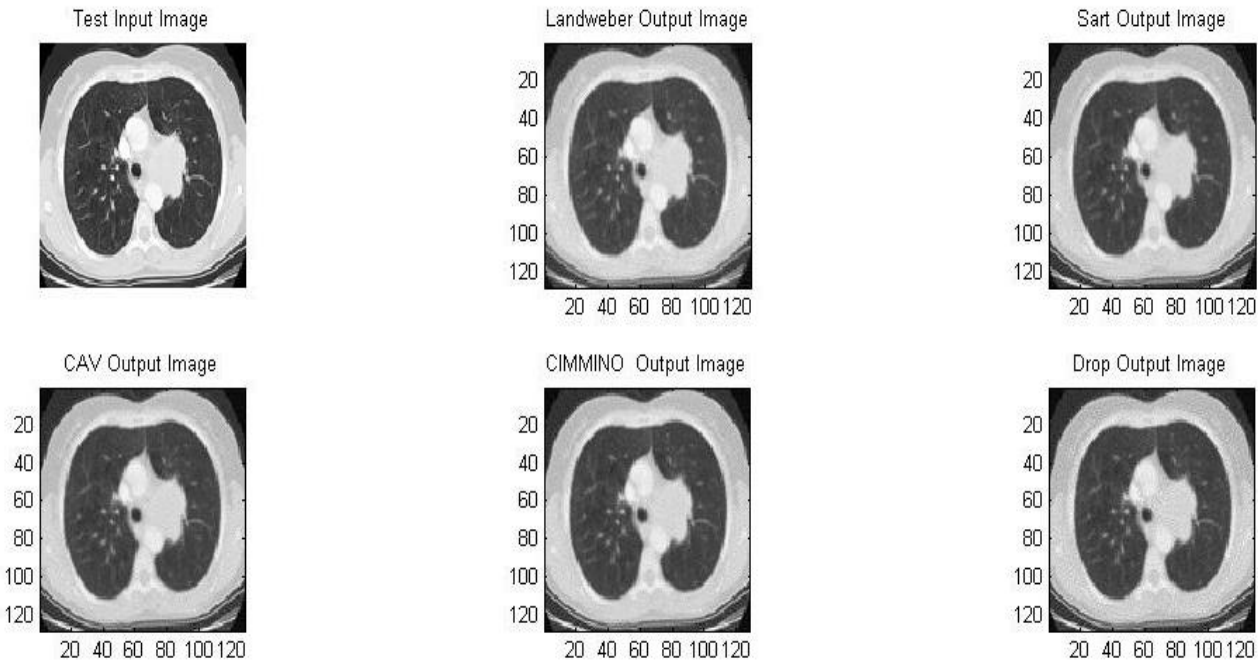

(c)

Figure 3. (a) Test Image and reconstructed image (b) Head image and reconstructed image (c) Thorax image and reconstructed image

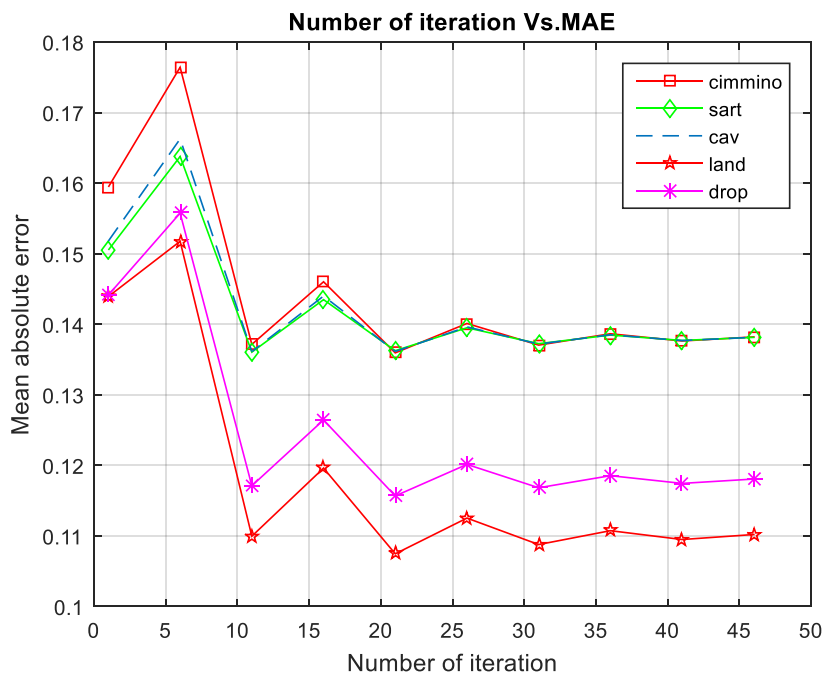

Figure 4. Mean absolute error between original and reconstructed image (Parallel beam - test image)

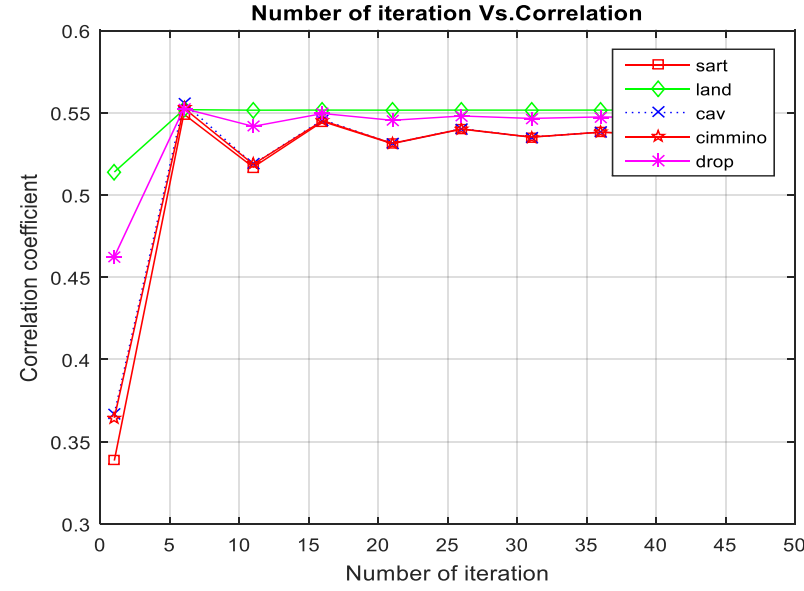

Figure 5. Correlation coefficient between original and reconstructed image (Parallel beam- test image) 


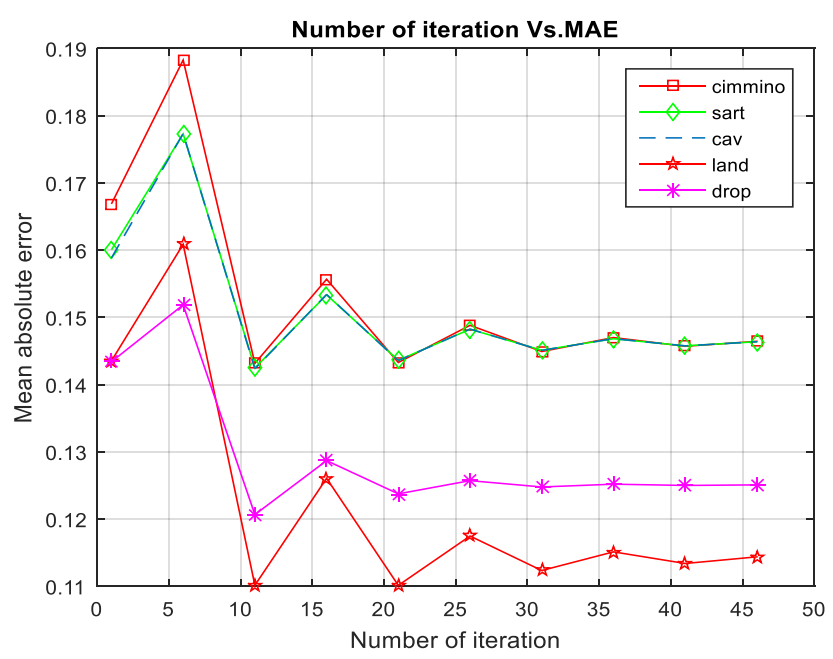

Figure 6. Mean absolute error between original and reconstructed image (Fan beam - test image)

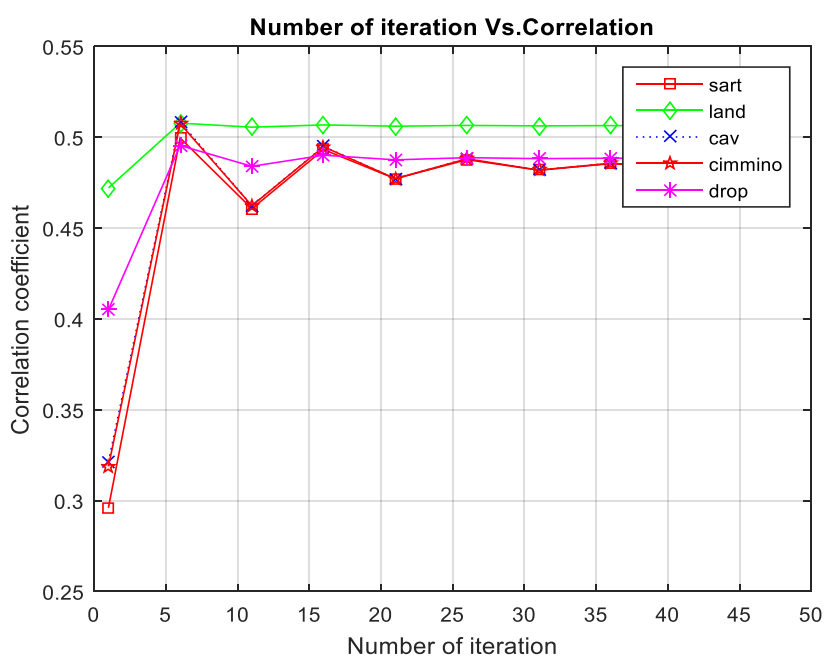

Figure 7. Correlation coefficient between original and reconstructed image (Fan beam - test image)

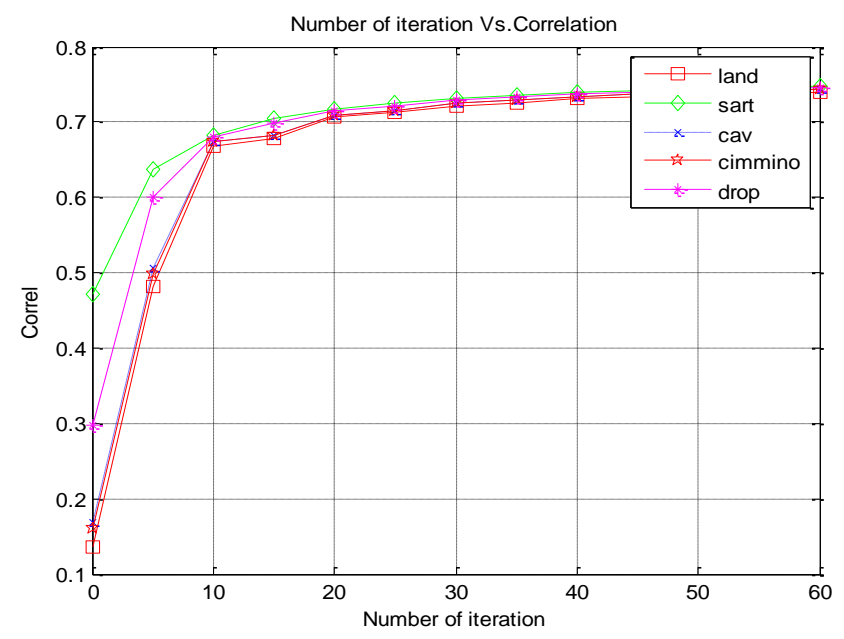

Figure 8. Correlation coefficient between original and reconstructed image (Fan beam - head image)

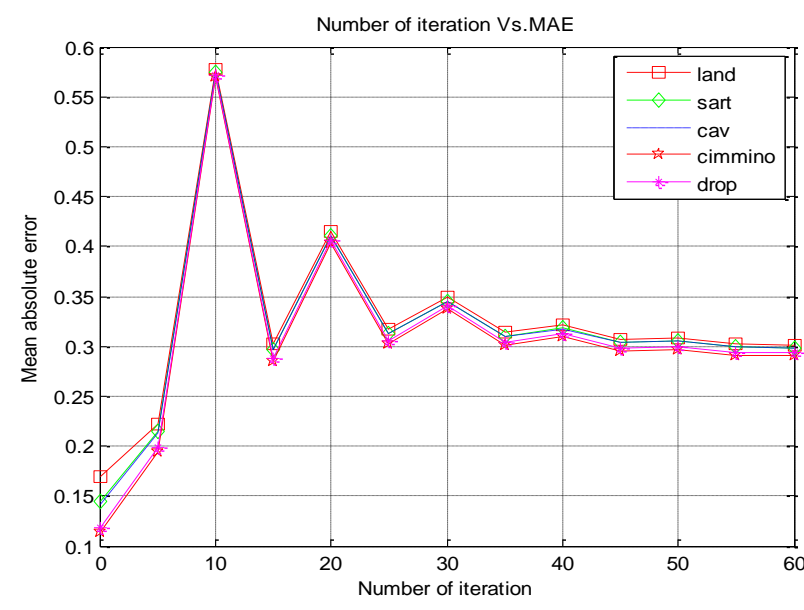

Figure 9. Mean absolute error between original and reconstructed image

(Fan beam - head image)

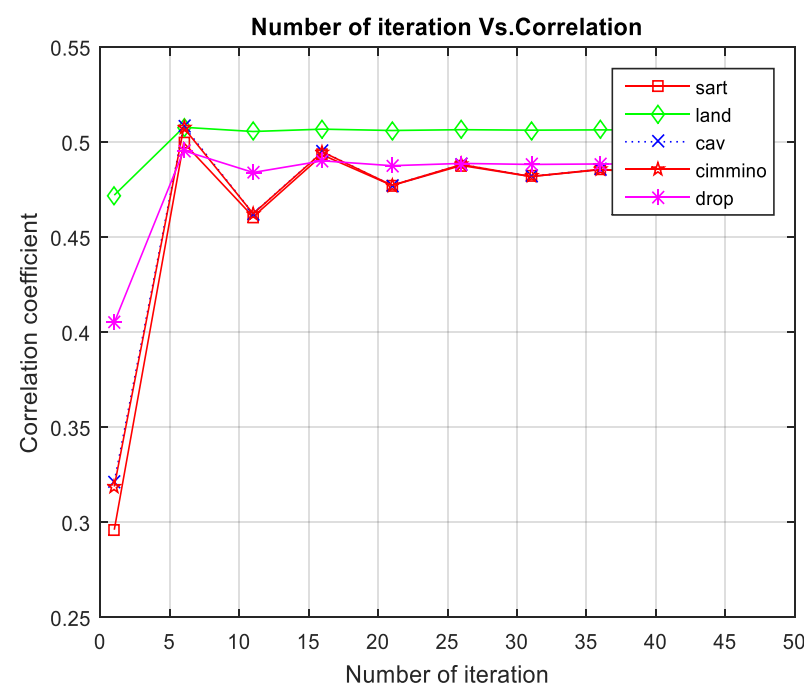

Figure 10. Correlation coefficient between original and reconstructed image

(Fan beam - thorax image)

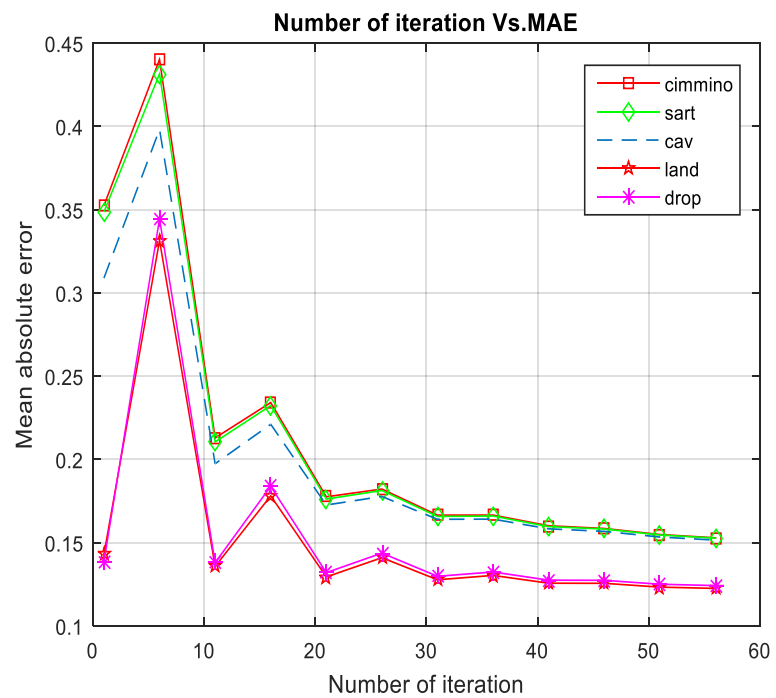

Figure 11. Mean absolute error between original and reconstructed image

(Fan beam - thorax image) 


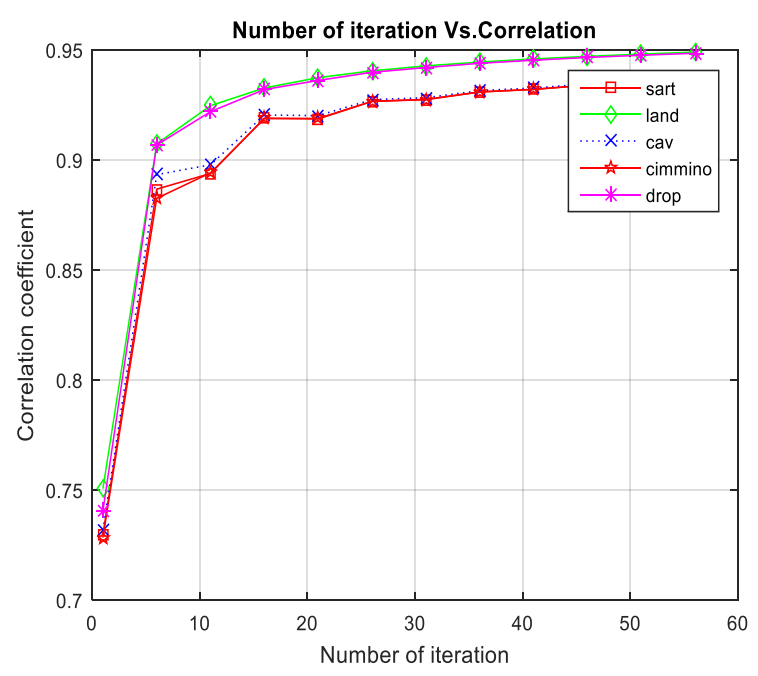

Figure 12. Correlation coefficient between original and reconstructed image

(Parallel beam - thorax image)

Figs. 4 to 7 show the performance comparison between proposed and other iterative algorithm. The mean square error and correlation coefficient are measured in original and reconstructed image for different iteration in Test phantom simulated with different iterative algorithms. The proposed landweber iteration algorithm produces much better results in lower iteration itself with quality of image.

Figs. 8 and Fig. 9 show the performance comparison of head image configured in parallel beam and fan beam tomography simulated with iterative algorithms and plot the curves of the proposed and other iterative algorithms. It can be observed that the reconstructed image of the proposed method is better than other algorithms. As lower number of iteration, less error is obtained between the original and reconstructed image with fixed number of projections.

Fig. 10 to Fig. 12 illustrate the mean square error and correlation coefficient is measured in thorax image in parallel and fan beam tomography simulated by the proposed and existing iterative algorithms.

Table 1 below shows the measurement of image quality by simulating different iterative algorithms in the Test Phantom, Head Image and Thorax Image and parameters like Structural Content (SC), Absolute Difference (AD), Maximum Difference (MD), Normalized Absolute Error (NAE), Normalized Cross Correlation (NCC), Mean Absolute Error (MAE) and Peak Signal to Noise Ratio (PSNR) are calculated.

There have been many attempts in simulation were taken to produce the good quality of image in less number of projections and iterations. It is observed that the proposed algorithm called landweber iteration algorithm proved to be good in visual perception and error analysis compared to other algorithms.

Table 1. Performance of proposed scheme

\begin{tabular}{|c|c|c|c|c|c|c|c|c|}
\hline $\begin{array}{c}\text { Image / } \\
\text { Parameters }\end{array}$ & $\begin{array}{c}\text { Reconstruction } \\
\text { Algorithm }\end{array}$ & SC & AD & NAE & MD & $\mathrm{NCC}$ & MAE & PSNR \\
\hline \multirow{5}{*}{$\begin{array}{c}\text { Test } \\
\text { Image } \\
\text { Fan Beam }\end{array}$} & $\begin{array}{l}\text { Landweber } \\
\text { (Proposed) }\end{array}$ & 2.18 & 0.014 & 0.884 & 0.995 & 0.488 & 0.98 & 9.636 \\
\hline & SART & 1.899 & 0.037 & 0.857 & 0.962 & 0.500 & 1.0 & 9.616 \\
\hline & CAV & 2.180 & 0.014 & 0.884 & 0.995 & 0.500 & 0.98 & 9.626 \\
\hline & CIMMNO & 2.180 & 0.014 & 0.885 & 0.995 & 0.458 & 0.96 & 9.624 \\
\hline & DROP & 1.963 & 0.003 & 0.884 & 0.991 & 0.489 & 0.96 & 9.626 \\
\hline \multirow{5}{*}{ Parallel Beam } & $\begin{array}{l}\text { Landweber } \\
\text { (Proposed) }\end{array}$ & 2.412 & 0.017 & 0.916 & 0.97 & 0.414 & 0.93 & 9.627 \\
\hline & SART & 2.016 & 0.0780 & 0.890 & 0.93 & 0.466 & 0.06 & 9.626 \\
\hline & CAV & 2.412 & 0.0177 & 0.917 & 0.94 & 0.467 & 0.07 & 9.624 \\
\hline & CIMMNO & 2.413 & 0.0176 & 0.915 & 0.95 & 0.414 & 0.06 & 9.623 \\
\hline & DROP & 2.101 & 0.087 & 0.916 & 0.96 & 0.449 & 0.06 & 9.625 \\
\hline \multirow{5}{*}{ Thora } & $\begin{array}{l}\text { Landweber } \\
\text { (Proposed) }\end{array}$ & 2.100 & 0.035 & 1.098 & 238.418 & 0.99 & 0.121 & 78.936 \\
\hline & SART & 2.101 & 0.032 & 1.098 & 238.431 & 0.399 & 0.120 & 78.935 \\
\hline & CAV & 2.101 & 0.032 & 1.090 & 238.431 & 0.399 & 0.121 & 78.924 \\
\hline & CIMMNO & 2.098 & 0.011 & 1.099 & 238.430 & 0.399 & 0.135 & 78.940 \\
\hline & DROP & 2.104 & 0.080 & 1.099 & 238.394 & 0.399 & 0.140 & 78.996 \\
\hline
\end{tabular}




\section{CONCLUSION}

Landweber based iterative reconstruction algorithm is implemented for computer tomography in parallel beam and cone beam to achieve the good quality of images in the reduced dose level in order to reduce the overall radiation exposure. The problems of imaging are dealt with the tradeoff between data size, the image quality and the algorithm computational complexity to create the image in reduced dose level. In this paper, SART, CAV, CIMMNO, DART and landweber iterative reconstruction algorithms are simulated in reduced dose level. The performances of execution are verified in terms of the quality of image. The reduction of dose is accomplished through drop off the tube current like 100 milli amps to 40 and 17 milli amps. The Landweber iterative reconstruction in the reduced dose level performs better image quality in different images.

\section{REFERENCES}

[1] Magrini A, Lazzari S, Marenco L, Guazzi G. (2017). A procedure to evaluate the most suitable integrated solutions for increasing energy performance of the building's envelope, avoiding moisture problems. International Journal of Heat and Technology 35(4): 689-699. https://doi.org/10.18280/ijht.350401

[2] Kim K. et al. (2015). Sparse-view spectral CT reconstruction using spectral patch-based low-rank penalty. IEEE Transactions on Medical Imaging 34(3): 748-760. https://doi.org/10.1109/TMI.2014.2380993

[3] Navitha CH, Sivani K, et al. (2017). Adaptive wavelet transform based rake receiver for ultra-wideband systems. AMSE-IIETA Journals -Series: Advances B 60(2): 357-371.

[4] Joshi S, Karule PT. (2018). Review of preprocessing techniques for fundus image analysis. AMSE-IIETA Journals-2017-Series: Advances B 60(2): 593-612.

[5] Wei HC, Li XB. (2017). Agent simulation model based on similarity measure. AMSE-IIETA Journals -2017Series: Advances D 60(4): 657-672.

[6] McCollough CH, Primak AN, et al. (2009). Strategies for reducing radiation dose in $\mathrm{CT}$. Radiological Clinical of North America 47(1): 27-40.

[7] Ravenel JG, Leue WM, et al. (2008). Pulmonary nodule volume: Effects of reconstruction parameters on automated measurements - a phantom study. Radiology 247(2): 400-408.

[8] $\mathrm{Xu} \mathrm{Q}, \mathrm{Yu} \mathrm{HY}$,et al. (2012). Low-dose x-ray CT reconstruction via dictionary learning. IEEE Transactions on Medical Imaging 31(9): 1682-1697. https://doi.org/10.1109/TMI.2012.2195669

[9] Needell D, Tropp JA. (2009). Iterative signal recovery from incomplete and inaccurate samples. Applied and Computational Harmonic Analysis 26(3): 301-321.

[10] Han X, Bian JG, et al. (2011). Algorithm-enabled lowdose micro-CT imaging. IEEE Transactions on Medical Imaging 30(3): 606-620. https://doi.org/10.1109/TMI.2010.2089695

[11] Zambelli J, Bevins N, et al. (2010). Radiation dose efficiency comparison between differential phase contrast $\mathrm{CT}$ and conventional absorption $\mathrm{CT}$. International journal of Medical Physics Letter 37: 2473-2479. https://doi.org/10.1118/1.3425785
[12] Dutta P. (2017). Medical diagnosis via distance measures on picture fuzzy sets. AMSE Journals AMSE IIETA publication-2017-Series: Advances A 54(2): 657-672.

[13] Yang CY, Pan SP. (2017). New practical determination of non-singular h-matrices. AMSE JOURNALS-AMSE IIETA publication-Series: Advances A 54(2): 373-383.

[14] Zhou J, Guo K, Ye S. (2015). The review of erroreliminating theories. AMSE JOURNALS-Series: Advances A 52(1):1-16.

[15] Yu L, Liu X, et al. (2009). Radiation dose reduction in computed tomography: Techniques and future perspectives. Imaging in Medicine 1(1): 65-84. https://doi.org/10.2217/iim.09.5

[16] Singh S, Karla MK, et al. (2011). Adaptive statistical iterative reconstruction techniques for radiation dose reduction in chest CT: A pilot study. Radiological Society of North America Journal 259(2) 565-573.

[17] Sodickson A. (2013). Strategies for reducing radiation exposure from multidetector computed tomography in the acute care setting. Canadian Association of Radiologists Journal 64: 119-129.

[18] Boas FE, Fleischmann D. (2012). CT artifacts: Causes and reduction techniques. IEEE Transaction on Medical Imaging 4(2): 29-240.

[19] Peng H, Stark H. (1989). One step image reconstruction from incomplete data in computer tomography. IEEE Transaction on Medical Imaging 8(1): 16-31. https://doi.org/10.1109/42.20358

[20] Guany H, Gordon R, et al. (1998). Combining various projection access schemes with the algebraic reconstruction technique for low-contrast detection in computed tomography. Physics of Medical and Biology 43: 2413-2421.

[21] Zhou JG, Ye QX, et al. (2014). Error elimination based on error set. AMSE Journals -2014-Series: Advances A 51(1): 13-26.

[22] Khamrui A, Mandal JK. (2014). A genetic algorithm based approach in image authentication using $Z$ transform (GAIAZT). AMSE Journals- Series: Advances B 57(1): 20-30.

[23] Ghosal SK, Mandal JK. (2014). Color image authentication based on two-dimensional separable discrete hartley transform (CIA2D-SDHT). AMSE Journals -Series: Advances B 57(1): 68-87.

[24] Mandal RK, Manna N. (2014). Handwritten english character recognition using hoof segmentation of image matrix (HSIM). AMSE Journals -Series: Advances B 57(1): 88-107.

[25] Aweda MA, Arogundade RA. (2007). Patient dose reduction methods in computerized tomography procedures: A review. International Journal of Physical Sciences 2(1): 001-009.

[26] Cervinkova I, Walek P, et al. (2016). Possibilities of reducing radiation dose in computed tomography examinations in various age groups using an iterative model-based reconstruction technique. Pediatrics \& Therapeutics 6(4): 2-7. https://doi.org/10.4172/21610665.1000302

[27] Scarfe WC, Farman AG. (2008). What is cone-beam CT and how does it work? The Dental Clinics of North America 52: 707-730.

[28] Toft PA, Sorensen JA. (1996). The radon transform theory and implementation. kgs. Lyngby, Denmark: 
Technical University of Denmark (DTU).

\section{NOMENCLATURE}

Algorithm

A precise set of steps to be performed in a specific order to solve a problem. Algorithms are the basis for most computer programming.

Attenuation The system accounts for the attenuation profile properties of each ray sum and correlates it to the position of the ray.

Back Process of converting the data from the projection Beam pitch

Clinical information systems (CIS)

Computed tomography dose index (CTDI) Computerize d physician order-entry (CPOE) Cone beam

CTDIvol

CTDIw

Detector

Detector aperture Detector array

Detector efficiency

Detector pitch

Detector spacing attenuation profile to a matrix.

Table movement per rotation divided by beam width.

Information systems that keep track of clinical data.

Collimators: Mechanical hardware that resembles small shutters and adjusts the opening based on the operator's selection.

Dose reported to the FDA; slices must be contiguous.

System that electronically transmits clinician orders to radiology and other

The radiation emitted from the collimated $\mathrm{x}$-ray source in multidetector row $\mathrm{CT}$ systems.

The CTDIvol is a measure of exposure per slice and is independent of scan length. It is the preferred expression of radiation dose in CT dosimetry.

The CTDIw adjusts for variation across the scan field of view by providing a weighted average of measurements at the center and the peripheral slice locations (i.e., the $\mathrm{x}$ and $\mathrm{y}$ dimensions of the slice).

Element in a CT system that collects attenuation information. It measures the intensity of the transmitted x-ray radiation along a beam projected from the $\mathrm{x}$-ray source to that particular detector element.

Size of the detector opening.

Entire collection of detectors included in a CT system; detector elements are situated in an arc or a ring.

Ability of the detector to capture transmitted photons and change them to electronic signals.

Table movement per rotation time divided by the selected slice thickness of the detector.

Measured from the middle of one detector to the middle of the departments. neighbouring detector; accounts for the spacing bar.
Effective dose

A measurement, reported in $\mathrm{Sv}$ or rem, that attempts to account for the effects particular to the patient's tissue that has absorbed the radiation dose. It extrapolates the risk of partial body exposure to patients from data obtained from whole body doses to Japanese atomic bomb survivors. Although methods to calculate the effective dose have been established, they depend on the ability to estimate the dose to radiosensitive organs from the $\mathrm{CT}$ procedure. Also called effective dose equivalent.

Effective Thickness of the slice that is actually slice represented on the CT image, as thickness opposed to the size selected by the collimator opening. In traditional axial scanning, selected slice thickness is equal to effective slice thickness However, because of the interpolation process used in helical scanning, the effective slice thickness may be wider than the selected slice thickness. Also called the slice-sensitivity profile.

Fan beam The radiation emitted from the collimated $x$-ray source in singledetector row CT systems.

Focal spot Area of the anode where the electrons strike and the $\mathrm{x}$-ray beam is produced.

Fourier

transform

(FT)

Gantry A method to study waves of many different sorts and also to solve several kinds of linear differential equations. Loosely speaking it separates a function into its frequency components.

Ring-shaped part of the CT scanner that houses many of the components necessary to produce and detect $\mathrm{X}$-rays

Gray scale System that assigns a certain number of Hounsfield values to each shade of gray.

High An x-ray beam is greatly impeded by an attenuation object; typically shown as light gray or white on an image.

High- Ability of a system to resolve, as contrast separate forms, small objects that are resolution very close together. Also call spatial resolution or detail resolution.

Image Anything appearing on the image that is artifacts not present in the object scanned.

Image Use of raw data to create an image.

reconstructio

$\mathrm{n}$

Isotropic

Kilovoltpeak $(\mathrm{kVp})$

Low

attenuation

Matrix

Milliampere Measure of the tube current used in the (mA) production of x-ray energy. In conjunction with the scan time, it is the 
Milliampere- The product of milliampere setting

seconds

(mAs)

Nonuniform Detector rows that have variable widths arrays

Organ dose

Pitch profile

Raw data

Ray

Ray sum

Reconstructi on algorithm

Reference

dose values

Reference

image

Region

interest

and sizes. Also called adaptive or hybrid arrays.

The estimated radiation dose to radio sensitive organs from CT procedures. These averages are used to calculate effective dose.

Relation of table speed to slice thickness. It is most commonly defined as the travel distance of the CT scan table per $360^{\circ}$ rotation of the $\mathrm{x}$-ray tube, divided by the $\mathrm{x}$-ray beam collimation width.

Variations along the length, or $\mathrm{z}$ axis, of the patient; also referred to as the $\mathrm{z}$-axis dose distribution.

All measurements obtained from the detector array and sitting in the computer waiting to be made into an image. Also called scan data.

The path that the x-ray beam takes from the tube to the detector.

The detector senses each arriving ray and senses how much of the beam was attenuated.

Determines how the data are filtered in the reconstruction process. The appropriate reconstruction algorithm selection depends on which parts of the data should be enhanced or suppressed to optimize the image for diagnosis.

Values published by the ACR regarding the radiation dose that is acceptable for a variety of CT scans.

Displays the slice lines in corresponding locations on the scout image.

of An area on the image defined by the operator.
(ROI):

Scan

parameters

thickness

Spatial resolution

Threshold

CT values

Tube current

Uniform

array

View

Voxel

$\mathrm{Z}$ axis
Factors that can be controlled by the operator and affect the quality of the image produced. These factors include milliamperes, scan time, slice thickness, field of view, reconstruction algorithm, and kilovolt-peak. When using helical scan methods, the operator also has a choice of pitch.

Time the $\mathrm{x}$-ray beam is on for the collection of data for each slice. Most often it is the time required for the gantry to make a $360^{\circ}$ rotation, although with over scanning and partial scanning options there may be some mild variation.

On a single-detector row system this is controlled by the width of the collimator opening. On a multidetector row system it is controlled by a combination of collimation and detector configuration.

Ability of a system to resolve, as separate forms, small objects that are very close together. Also call high contrast resolution or detail resolution.

A predetermined CT value limit set by the operator in some types of 3D reformation techniques. The software will include or exclude the voxel depending on whether its CT number is above or below the threshold.

Measured in thousandths of an ampere, or milliamperes, it controls the quantity of electrons propelled from cathode to anode.

Detector rows that are parallel and of equal size.

A complete set of ray sums.

Volume element. Three-dimensional cube of data acquired in $\mathrm{CT}$.

Plane that correlates to the slice thickness, or depth, of the CT slice. 